Pesq. Vet. Bras. 30(7):566-572, julho 2010

\title{
Biópsia pulmonar incisional por toracoscopia paraxifoide transdiafragmática com dois portais em cães ${ }^{1}$
}

\author{
Paula C. Basso ${ }^{2 \star}$, Alceu G. Raiser ${ }^{3}$, Maurício V. Brun ${ }^{4}$, Nédio Guizzo Junior ${ }^{5}$, \\ João P.S. Feranti ${ }^{6}$, Adriana C. Motta ${ }^{4}$, Carlos E. Bortolini ${ }^{7}$ e Daniel C.M. Muller ${ }^{2}$ \\ ABSTRACT.- Basso P.C., Raiser A.G., Brun M.V., Guizzo Junior N., Feranti, J.P.S., Motta, \\ A.C., Bortolini, C.E. \& Muller D.C.M. 2010. [Pulmonary incisional biopsy by means of \\ transdiaphragmatic paraxiphoid thoracoscopy with two ports in dog.] Biópsia pulmonar \\ incisional por toracoscopia paraxifoide transdiafragmática com dois portais em cães. Pesquisa \\ Veterinária Brasileira 30(7):566-572. Departamento de Clínica de Pequenos Animais, Centro \\ de Ciências Rurais, Universidade Federal de Santa Maria, Santa Maria, RS 97119-900, \\ Brazil. E-mail: paula.basso@bol.com.br \\ There are many respiratory diseases in small animals, as well as the possibilities of diagnoses, \\ as laboratory and radiographic exams, bronchoalveolar lavage and biopsies. This research aimed \\ at assessing the results of transdiaphragmatic paraxiphoid thoracoscopies in pulmonary biopsies \\ of thirteen clinically healthy dogs. Under general anesthesia, each dog was insufflated with $30 \mathrm{ml}$ \\ $\mathrm{kg}^{-1}$ of air in each hemithorax; two trocars were placed between the xiphoid appendix and the \\ costal arch through the diaphragm. An endoscope was introduced by the first portal; while the \\ second portal was used to allow access to the nippers in the biopsy forceps. Then, these nippers \\ were removed and replaced by chest tube drainage. During the procedures, respiratory frequency, \\ heart rate, oxygen saturation, arterial pressure and central venous pressure (CVP), hemogasometric \\ parameters were checked. Within 48 hours after the surgery, drains were or drain was removed, \\ with minimal production of air and/or liquid. In conclusion, this is a fast and safe technique, which \\ is free of trans and post surgical complications, thus allowing the collection of sufficient material \\ for pulmonary histological evaluation.
}

INDEX TERMS: Video-assisted thoracic surgery, chest, biopsy, respiratory diseases, dog.

RESUMO.- As doenças respiratórias observadas na clínica médica de pequenos animais são numerosas, assim como as possibilidades diagnósticas, dentre as quais en-

\footnotetext{
1 Recebido em 17 de março de 2009.

Aceito para publicação em 10 de março de 2010.

2 Pós-graduando de Doutorado em Medicina Veterinária (PPGMV), Centro de Ciências Rurais (CCR), Universidade Federal de Santa Maria (UFSM), Santa Maria, RS. Endereço particular: Rua São Boaventura 31, Bairro São Geraldo, ljuí, RS 98700-000, Brasil. *Autor para correspondência: paula.basso@bol.com.br

${ }^{3}$ Docente do Departamento de Clínica de Pequenos Animais (DCPA), CCR, UFSM, Av. Roraima 1000, Santa Maria, RS 97105-900, Brasil.

${ }^{4}$ Docente do Curso de Medicina Veterinária (CMV) da Faculdade de Agronomia e Medicina Veterinária, Universidade de Passo Fundo (UPF), BR 285 Km171, Passo Fundo, RS 99001-970, Brasil.

${ }^{5}$ Médico Veterinário do Hospital Veterinário, UPF, Passo Fundo, RS.

${ }^{6}$ Graduando do Curso de Medicina Veterinária, UPF, Passo Fundo, RS.

7 Pós-graduando de Mestrado em Medicina Veterinária (PPGMV), CCR, Universidade Federal do Rio Grande do Sul (UFRGS), Av. Paulo Gama 110, Porto Alegre, RS 90040-060, Brasil.
}

contram-se os exames laboratoriais, os estudos radiográficos, os lavados broncoalveolares e as biópsias. $\mathrm{Na}$ presente pesquisa avaliaram-se os resultados da toracoscopia paraxifoide transdiafragmática para a realização de biópsia pulmonar em cães, sendo utilizados para tanto, 13 animais clinicamente sadios. Sob anestesia geral, produziu-se pneumotórax no volume de $30 \mathrm{ml} \mathrm{kg}^{-1}$ de ar ambiente para cada hemitórax. O acesso foi obtido a partir de dois trocartes, posicionados entre o apêndice xifoide e o arco costal, os quais transfixaram o diafragma. O primeiro portal foi empregado para a passagem do endoscópio e o segundo para a utilização de pinça saca-bocado, empregada na obtenção de biópsia. Em seguida, a pinça foi removida e um dreno torácico foi posicionado através do portal. Durante os procedimentos, aferiram-se as frequências respiratória e cardíaca, a saturação de oxigênio, as pressões arterial média e venosa central e os parâmetros hemogasométricos. Os drenos foram removidos num período de até 48 horas de pós-operatório, verificando-se mínima produção de ar e/ou líquido. Concluiu-se se tratar de 
uma técnica rápida, segura e sem complicações trans e pós-operatórias, permitindo aquisição de material suficiente para a avaliação histológica do pulmão.

TERMOS DE INDEXAÇÃO: Cirurgia torácica vídeo-assistida, tórax, biópsia, doença respiratória, cães.

\section{INTRODUÇÃO}

Distúrbios do parênquima pulmonar são rotineiramente diagnosticados em cães e gatos. As pneumonias infecciosas e neoplasias são causas comuns de doenças parenquimatosas, entretanto as doenças pulmonares intersticiais são raras e representam um desafio diagnóstico na clínica de pequenos animais. Dentro dessa classe encontram-se a pneumonia eosinofílica, a fibrose pulmonar intersticial, a pneumonia intersticial linfocítica, e a pneumonia lipídica endógena, entre outras (Reineiro \& Cohn 2007).

A radiografia torácica, por ser método não invasivo e de custo acessível, é o exame complementar mais utilizado nas afecções pulmonares. No entanto, o exame radiográfico torácico fornece padrões de alterações por vezes inespecíficos, limitando o diagnóstico (Norris et al. 2002). Por outro lado, o lavado broncoalveolar fornece dados citológicos e microbiológicos que permitem uma melhor definição do distúrbio respiratório. Contudo, Andreasen (2003) ratifica que a análise desse fluido ainda pode ser insuficiente para o diagnóstico definitivo, pois os achados da lavagem broncoalveolar de cães foram considerados definitivos em apenas $25 \%$ dos casos.

Quando as radiografias torácicas e as análises citológicas e microbiológicas não promoverem o diagnóstico definitivo, torna-se necessária a realização de biópsia pulmonar e do exame histopatológico (Norris et al. 2002). A colheita de material para biópsia pulmonar pode ser efetuada através de broncoscopia flexível, a qual permite a visibilização interna das estruturas do trato respiratório e possibilita aquisição de amostras de fluido broncoalveolar e de tecido pulmonar em locais específicos (Miller 2007). Entretanto, um dos problemas bem reconhecidos da biópsia pulmonar transbrônquica é a falta de tecido parenquimatoso representativo, ou seja, em aproximadamente $50 \%$ dos casos pode existir apenas a presença de parede bronquial (Colt et al. 1995).

A toracotomia ou a toracoscopia, como oposto a broncoscopia, permitem a obtenção de maiores amostras de tecido pulmonar sob visualização direta (Harris et al. 1995), e podem ser aplicadas em instituições que não dispõem de endoscopia flexível. Técnicas abertas têm sido consideradas efetivas para a obtenção de biópsia pulmonar, no entanto, por tratar-se de uma abordagem cirúrgica agressiva, a morbidade e mortalidade no trans e pós-operatório aumentam significativamente (Rena et al. 1999). Em contrapartida, as toracoscopias são acessos minimamente invasivos, os quais reduzem o traumatismo e permitem adequada exposição cirúrgica sem reduzir a qualidade do procedimento. Além do mais, possibilita rápida recuperação do paciente e reduzida dor pós-operatória (Potter \& Hendrickson 1998).
Ressalta-se que a obtenção de biópsia pulmonar toracoscópica em cães, ainda é pouco explorada e geralmente requer a utilização de dois ou três portais (Potter e Hendrickson 1998). Dentre as técnicas de três portais, as mais comumente descritas consistem na aplicação de uma ligadura do parênquima pulmonar do tipo endoloop (Potter e Hendrickson 1998) e a ressecção de fragmento pulmonar em cunha com auxílio de sutura mecânica (Zoppa et al. 2008). Já as técnicas relatadas utilizando-se dois portais incluem a ressecção em cunha empregando grampeadores endoscópicos (Colt et al. 1995) e biópsias com fórceps conectado a um eletrocautério (Boutin et al. 1982). Essas últimas, ainda que menos invasivas, apresentam a desvantagem do alto custo dos instrumentos e a necessidade de intubação seletiva (Colt et al.1995).

Dentre os acessos descritos para toracoscopia encontram-se o cervical, o intercostal e o paraxifoide transdiafragmático (Potter \& Hendrickson 1998). Esse último é descrito para procedimentos de pericardiectomia parcial em pacientes que apresentam efusão pericárdica. Possui vantagem sobre os demais, pois permite ampla exploração da cavidade torácica em ambos os hemitóraces e ótima visibilização do hilo pulmonar, mediastino e estruturas ventrais do tórax. Segundo alguns autores, os demais acessos permitem somente a exploração do hemitórax correspondente, podendo acarretar atraso na identificação de lesões que estão localizadas no hemitórax contralateral (Potter \& Hendrickson 1998, Twedt 2002).

Considerando a importância das doenças respiratórias em cães e o uso cada vez maior da cirurgia de acesso mínimo, este estudo busca avaliar o acesso toracoscópico paraxifoide transdiafragmático para colheita de biópsia pulmonar com pinça saca-bocado em cães. Justifica-se tal investigação pela condição de algumas instituições nacionais não disporem de broncoscopia flexível, mas possuírem aceso a equipamentos endoscópicos, e também pelo fato da biópsia transbrônquica nem sempre fornecer amostras teciduais representativas e em virtude do referido acesso toracoscópico possibilitar a colheita de amostras tissulares de ambos os pulmões.

\section{MATERIAL E MÉTODOS}

Foram utilizadas 13 cadelas, sem raça definida e com peso corporal médio de $13.69 \pm 5.14 \mathrm{~kg}$. Previamente à inclusão no experimento, os animais foram avaliados clinicamente, desverminados e alojados em canis individuais onde receberam ração comercial e água à vontade. No período pré-operatório, foram realizados hemograma completo e contagem plaquetária de todos os animais. Em seis cães, coletou-se sangue da artéria femoral com seringas heparinizadas, que foram adequadamente vedadas para posterior análise hemogasométrica.

O experimento foi dividido em duas etapas. Na primeira, realizou-se a colheita de biópsia pulmonar através do acesso cirúrgico toracoscópico paraxifoide transdiafragmático com dois portais. A segunda foi realizada 21 dias após, sendo os animais submetidos ao acesso toracoscópico intercostal para avaliação pós-operatória. 
Os animais foram submetidos a jejum alimentar de 12 horas e pré-medicados com acepromazina a 0,2\% $\left(0,05 \mathrm{mg} \mathrm{kg}^{-1}\right.$, IM) e sulfato de morfina $\left(0,5 \mathrm{mg} \mathrm{kg}^{-1}, \mathrm{IM}\right)$. Após 15 minutos, foram induzidos com tiopental sódico (12mg kg-1, IV) e a manutenção anestésica foi obtida com isofluorano vaporizado em oxigênio a $100 \%$, em circuito semifechado, com respiração assistida.

Posteriormente, cada animal foi posicionado em decúbito dorsal, sendo introduzido um cateter venoso periférico (18G) na veia jugular direita para a mensuração da pressão venosa central. Este foi heparinizado e fixado à pele com pontos isolados simples com náilon monofilamentar 3-0. Os equipos para mensuração da Pressão Venosa Central (PVC) foram montados em haste de suporte para fluidos, e uma régua com escala em centímetros, foi posicionada com o seu ponto zero em nível do átrio direito. O nível para a fixação do ponto zero foi estabelecido por meio da palpação do manúbrio, sendo utilizada uma régua nivelada para evitar quaisquer desvios. Após a mensuração aplicou-se o fator de correção $-0,51 \mathrm{cmH}_{2} \mathrm{O}$ nos valores da leitura da PVC (Aguiar et al. 2004).

Para a aferição da pressão arterial média (PAM) e colheita de sangue arterial, foi realizada a canulação da artéria femoral esquerda com cateter periférico (20G), conectado com dois extensores de equipo intercalados por uma torneira de três vias, permitindo a heparinização periódica. O último extensor estava acoplado a um manômetro de mercúrio, sendo a parte inferior deste nivelada com o ponto zero da PVC.

Em seguida, procedeu-se a anti-sepsia bilateral do tórax e do abdome cranial. Os animais foram submetidos a uma toracocentese com escalpe ( $n-21$ ) no sétimo espaço intercostal bilateralmente e, com o auxílio de uma seringa de $60 \mathrm{ml}$ e uma torneira de três vias, foram introduzidos $30 \mathrm{ml} \mathrm{kg}^{-1}$ de ar ambiente em cada hemitórax, fracionados em três aplicações subsequentes. Após 5 minutos, realizou-se incisão de pele de aproximadamente $0,5 \mathrm{~cm}$ entre o apêndice xifoide e o arco costal, por onde foi introduzido um trocarte de $5 \mathrm{~mm}$ de diâmetro, o qual percorreu um trajeto subcutâneo de aproximadamente $2 \mathrm{~cm}$. A cânula, após o trajeto, foi direcionada craniolateralmente através do diafragma (Fig.1) no hemitórax direito. Com a torneira de insuflação da cânula mantida aberta, introduziu-se um endoscópio rígido de $5 \mathrm{~mm}$ de diâmetro. Quando necessário, perfurou-se a membrana mediastínica com o próprio instrumento, procedendo-se também a exploração do hemitórax contralateral.

Na sequência, foi repetido o mesmo procedimento com o segundo trocarte, dessa vez no hemitórax esquerdo, no qual foi inserida outra cânula de $5 \mathrm{~mm}$ e uma pinça para biópsia

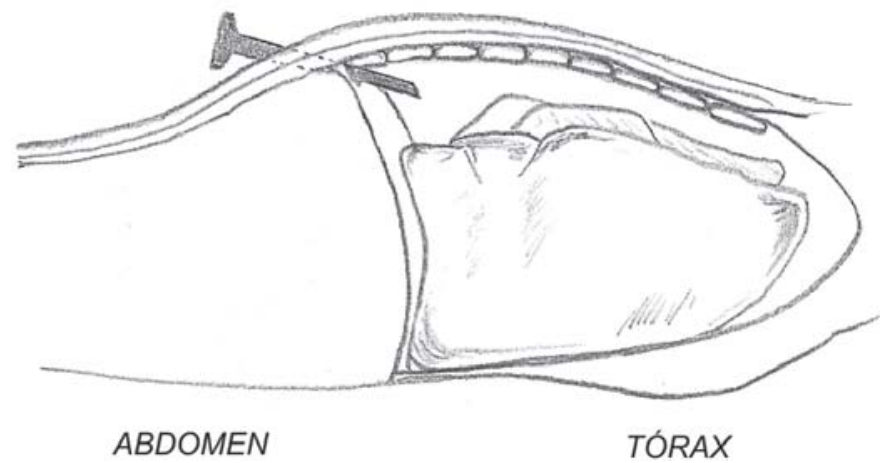

Fig.1. Imagem representativa do posicionamento do trocarte transdiafragmático para realização de biópsia pulmonar incisional em cão (Vista lateral). saca bocado de mesmo diâmetro. Depois de adequada inspeção de ambos os pulmões, realizou-se o pinçamento do lobo cranial direito e, com a introdução intercostal de uma agulha $40 \times 12 \mathrm{~mm}$, foi possível apoiar o pulmão contra a pleura parietal e seccionar um fragmento tissular de aproximadamente $0,5 \mathrm{~cm}$. Em seguida, retirou-se a pinça e procedeu-se a insuflação pulmonar para certificar-se da ausência de escape de ar. Um dreno de tórax (10Fr) foi posicionado através do segundo trocarte de $5 \mathrm{~mm}$, sem a válvula, direcionado ao hemitórax correspondente à lesão. $O$ trocarte foi removido, a pressão torácica negativa foi restituída, e o local de punção na pele foi obliterado com fio de náilon monofilamentar 4-0 em padrão isolado simples. Já o dreno foi fixado com fio de seda 2-0 por ponto chinês. O material da biópsia foi identificado e fixado em formol, sendo posteriormente incluído em parafina, corado pela hematoxilina eosina, e avaliado por microscopia óptica.

As medidas da frequência cardíaca ( $F C)$, frequência respiratória (FR), saturação de oxigênio $\left(\mathrm{SpO}_{2}\right)$, PAM e PVC foram tomadas em cinco momentos: Após a indução da anestesia (M1), após a indução do pneumotórax (M2), posteriormente à entrada dos trocartes (M3), após a realização da biópsia (M4) e após o restabelecimento da pressão torácica negativa (M5). Já os parâmetros hemogasométricos do sangue arterial $(\mathrm{pH}$; pressão parcial do dióxido de carbono $\left[\mathrm{pCO}_{2}\right.$; pressão parcial de oxigênio $\left[\mathrm{pO}_{2}\right]$. bicarbonato $\left[\mathrm{HCO}_{3}^{-}\right]$; dióxido de carbono total $\left[\mathrm{CO}_{2}\right.$ Total]; saturação de oxigênio; sódio; potássio e lactato) foram analisados em três momentos: Antes da medicação préanestésica (M0), posteriormente a entrada dos trocartes (M3) e após a restituição da pressão negativa (M5).

Ao término do procedimento, os animais foram medicados com cloridrato de tramadol (3mg kg-1 $, \mathrm{SC}, \mathrm{TID}$, durante dois dias) e cetoprofeno (1 $\mathrm{mg} \mathrm{kg}^{-1}$, SC, SID, durante três dias). A drenagem torácica foi efetuada no pós-operatório com seringa de $60 \mathrm{ml}$, a cada 4 horas, e o dreno torácico foi removido quando a quantidade de ar e/ou líquido produzida foi inferior à $2,2 \mathrm{ml} \mathrm{kg}^{-1} \mathrm{dia}^{-1}$.

Para a segunda etapa do experimento, os animais foram submetidos à mesma sedação e anestesia previamente mencionadas. Após o posicionamento em decúbito dorsal e realização de anti-sepsia na região torácica direita, uma incisão de aproximadamente $0,5 \mathrm{~cm}$ foi feita no 6 o espaço intercostal, abrangendo a pele, o tecido subcutâneo e a musculatura. Pela ferida, foi introduzido o trocarte de $5 \mathrm{~mm}$ e em seguida, o endoscópio rígido foi acoplado iniciando a inspeção da cavidade torácica, enfatizando o sítio de biópsia e a região diafragmática de acesso. Após a remoção do trocarte, o orifício foi suturado com um único ponto isolado simples com náilon monofilamentar 3-0, envolvendo a pele, subcutâneo e camada muscular. A pressão torácica negativa foi restabelecida por toracocentese no oitavo espaço intercostal com scalpe número 21.

Foram aferidas as $\mathrm{FC}, \mathrm{FR}, \mathrm{SpO}_{2}, \mathrm{PAM}, \mathrm{PVC}$ e as variáveis hemogasométricas nos diferentes momentos da primeira etapa de experimentação. Tais medidas foram analisadas pelo teste estatístico Bonferroni, considerando as variações significativas quando $p<0,05$. Para verificar a qualidade da amostra obtida por biópsia, realizou-se a avaliação histopatológica do material coletado. Já a eficácia da biópsia pulmonar por toracoscopia paraxifoide transdiafragmática foi analisada por meio do tempo cirúrgico, ausência de complicações, drenagem torácica eficiente no período pós-operatório e completo selamento do defeito diafragmático, observado na reavaliação toracoscópica intercostal. O experimento foi aprovado pelo 
comitê de ética em pesquisa (CEP) da Universidade de Passo Fundo (UPF), sob registro 049/2008, e seguiu as normas de bem-estar preconizadas pelo COBEA (Colégio Brasileiro de Experimentação Animal).

\section{RESULTADOS E DISCUSSÃO}

Em todos os animais foi possível realizar o exame proposto. O procedimento de colheita de biópsia pulmonar durou $18,08 \pm 3,65$ minutos da incisão ao fechamento da ferida, sendo considerado substancialmente curto em relação às técnicas convencionais de biopsia por toracotomia. Segundo Bomback (2007), as toracotomias despendem maior tempo devido à ampla exposição tecidual e ao prolongado período para a realização das suturas.

Em relação aos dados de $\mathrm{FC}, \mathrm{SpO}_{2}$ e PAM obtidos durante o procedimento cirúrgico, não foi evidenciada diferença significativa entre os momentos avaliados (Quadro 1), o que associado aos resultados cirúrgicos, advoga quanto à segurança da técnica testada. No entanto, há de se considerar que após a indução do pneumotórax, foi necessária instituir ventilação assistida até o restabelecimento da pressão torácica negativa, a fim de se evitar qualquer queda brusca da $\mathrm{SpO}_{2}$ e a ocorrência de hipoxemia. A ventilação assistida é exigida também nas toracotomias para evitar o colabamento alveolar, que quando crônico, pode desencadear edema pulmonar após reexpansão, devido à liberação de radicais superóxidos (Fossum 2002).

A respeito dos valores aferidos da pressão venosa central, foi possível perceber que antes da indução do pneumotórax (M1), estes se encontravam dentro dos padrões normais citados por Aguiar et al. (2004), que variam de -2 a $4 \mathrm{cmH}_{2} \mathrm{O}$. Após a indução do pneumotórax (M2), ficou evidente a elevação significativa da PVC. Por fim, restaurando-se a pressão negativa intratorácica, houve uma di- minuição significativa nestes valores (M5), retornando próximos aos valores de M1 (Quadro 1). Isso é justificado uma vez que o aumento da pressão intratorácica é um determinante básico da PVC já que restringe o retorno venoso ao coração, comprometendo a dinâmica circulatória (Aguiar et al. 2004). Mesmo com o considerável aumento na PVC durante a execução da técnica, esses valores ainda estariam dentro dos limites normais $\left(0-10 \mathrm{cmH}_{2} \mathrm{O}\right)$ defendidos por Hendrix \& Raffe (1998), fato também mencionado por Pigatto et al. (2008) que encontraram sinais de descompensação de pneumotórax, apenas quando os valores atingidos foram iguais ou superiores a $10 \mathrm{cmH}_{2} \mathrm{O}$.

Os valores de $\mathrm{pH}, \mathrm{pCO}_{2}$ e $\mathrm{HCO}_{3}{ }^{-}$, verificados nas análises hemogasométricas pré-operatórias, encontravam-se dentro dos padrões considerados fisiológicos por Ochoa (2005), o que demonstra que anteriormente ao procedimento os pacientes estavam em situação hemodinâmica estável. De fato, as mensurações obtidas no transoperatório e após o termino da cirurgia, acusaram aumento significativo da $\mathrm{pCO}_{2}(\mathrm{M} 2 \mathrm{e}$ M3) e $\mathrm{HCO}_{3}^{-}(\mathrm{M} 3)$ com concomitante diminuição significativa do $\mathrm{pH}$ sanguíneo (M3 e M5), confirmando o desenvolvimento de acidose respiratória (Hopper \& Haskins 2008) que se prolongou mesmo ao término do procedimento (Quadro 1). Isso demonstra que a ventilação assistida foi insuficiente para a adequada oxigenação e, em virtude disso, a frequência respiratória aumentou significativamente (M5) na tentativa de compensar a hipoventilação (Quadro 1). Tal situação poderia ter sido evitada instituindo-se ventilação mecânica no transoperatório (Barreto et al. 2000).

A opção pelo acesso paraxifoide transdiafragmático nesse procedimento foi válida, pois permitiu ampla exploração da cavidade torácica em ambos os hemitóraces e excelente visibilização do parênquima pulmonar, fato defendido por Twedt (2002). Este acesso pode ser vantajoso

Quadro 1. Valores da média e desvio padrão da Frequência Cardíaca (FC), Frequência Respiratória (FR), Saturação de Oxigênio $\left(\mathrm{SpO}_{2}\right)$, Pressão Arterial Média (PAM), Pressão Venosa Central (PVC), pH arterial (pH), Pressão parcial do dióxido de carbono $\left(\mathrm{pCO}_{2}\right)$, Pressão parcial de oxigênio $\left(\mathrm{pO}_{2}\right)$, Bicarbonato $\left(\mathrm{HCO}_{3}{ }^{-}\right)$, Dióxido de carbono total $\left(\mathrm{CO}_{2}\right.$ Total), Saturação de oxigênio no sangue arterial ( $\left(\mathrm{SatO}_{2}\right)$,' Sódio, Potássio e Lactato, aferidos nos diferentes momentos $(\mathrm{MO}=$ antes da medicação pré-anestésica; $\mathrm{M1}$ = após a indução anestésica; M2 = após a indução do pneumotórax; M3 = após a entrada dos trocartes; M4 = após a execução da biópsia; M5 = após restituição da pressão intratorácica negativa)

\begin{tabular}{|c|c|c|c|c|c|c|}
\hline & M 0 & M 1 & M2 & M 3 & M 4 & M 5 \\
\hline $\mathrm{FC}(\mathrm{bpm})$ & _ & $117.15 .57 \pm 19.81$ & $122.15 \pm 26.11$ & $122.00 \pm 23.78$ & $120.69 \pm 28.69$ & $120.85 \pm 24.21$ \\
\hline FR (mpm) & - & $14.23 \pm 3.60$ & $20.46 \pm 9.38$ & $20.46 \pm 10.96$ & $20.31 \pm 12.14$ & $22.62 \pm 13.83^{*}$ \\
\hline $\mathrm{SpO}_{2}(\%)$ & - & $96.77 \pm 1.83$ & $94.77 \pm 3.76$ & $91.85 \pm 6.37$ & $94.38 \pm 3.31$ & $95.15 \pm 3.34$ \\
\hline PAM (mmHg) & - & $83.23 \pm 16.90$ & $85.77 \pm 16.77$ & $91.61 \pm 19.03$ & $97.15 \pm 16.16$ & $90.54 \pm 19.59$ \\
\hline PVC $\left(\mathrm{cmH}_{2} \mathrm{O}\right)$ & $\begin{array}{l}- \\
-\end{array}$ & $1.37 \pm 1.36$ & $3.54 \pm 1.62^{\star \star}$ & $4.47 \pm 1.63$ & $4.38 \pm 0.96$ & $1.18 \pm 1.14$ \\
\hline $\mathrm{pH}$ & $7.37 \pm 0.08$ & - & - & $7.21 \pm 0.04^{*}$ & - & $7.13 \pm 0,04$ \\
\hline $\mathrm{pCO}_{2}(\mathrm{mmHg})$ & $31.95 \pm 1.34$ & - & - & $56.83 \pm 5.80^{*}$ & - & $74.42 \pm 11.35^{*}$ \\
\hline $\mathrm{pO}_{2}(\mathrm{mmHg})$ & $83.53 \pm 7,96$ & - & - & $155.83 \pm 122.20$ & - & $238.22 \pm 108.99$ \\
\hline $\mathrm{HCO}_{3}^{-}(\mathrm{mmol} / \mathrm{L})$ & $18.25 \pm 0.49$ & - & - & $22.37 \pm 1.70^{*}$ & - & $24.05 \pm 1.71$ \\
\hline $\mathrm{CO}_{2}$ total $(\mathrm{mmol} / \mathrm{L})$ & $19.25 \pm 0.49$ & - & - & $24.10 \pm 1.79^{*}$ & - & $26.33 \pm 1.99$ \\
\hline Excessode base $(\mathrm{mmol} / \mathrm{L})$ & $-5.4 \pm 0.35$ & - & - & $-5.93 \pm 1.89$ & - & $-6.93 \pm 1.31$ \\
\hline $\mathrm{SatO}_{2}(\%)$ & $95.82 \pm 1.03$ & - & - & $95.82 \pm 3.29$ & - & $98.95 \pm 1.08$ \\
\hline Sódio (mEq/L) & $145.25 \pm 1.71$ & - & - & $147.67 \pm 2.58$ & - & $148.50 \pm 1.76$ \\
\hline Potássio (mEg/L) & $4.1 \pm 0.41$ & - & - & $3.73 \pm 0.26$ & - & $3.75 \pm 0.22$ \\
\hline Lactato $(\mathrm{mEq} / \mathrm{L})$ & $2 \pm 0.48$ & - & - & $2 \pm 0.44$ & - & $1.46 \pm 0.31$ \\
\hline
\end{tabular}

\footnotetext{
* Significativamente diferente $(p<0,05) ;{ }^{* *}$ significativamente diferente $(p<0,001)$.
} 
em relação ao toracoscópico intercostal, pois permite a aquisição de biópsia de qualquer lobo pulmonar de ambos os pulmões, condição muito importante em animais portadores de doenças respiratórias. Além disso, os autores desse trabalho consideram que o acesso toracoscópico intercostal seria mais traumático que o proposto, em virtude da existência de agressão à musculatura intercostal, da possível compressão dos nervos e vasos intercostais, e do risco de lesões dessas estruturas. Outro aspecto é que a musculatura está intimamente relacionada à expansão do tórax durante a inspiração, condição que poderia acarretar maior dor pós-operatória em relação ao acesso paraxifoide. Em relação ao acesso toracocópico cervical, considerou-se que o acesso paraxifoide pode ser mais seguro, uma vez que evitaria danos à artéria e veia torácica interna, ao tronco braquiocefálico e a artéria carótida comum, que podem ser complicações graves do acesso cervical, conforme afirmam Potter \& Hendrickson (1998).

Ao conhecimento do autor, a utilização de dois trocartes simultâneos pelos acessos paraxifoide direito e esquerdo, ainda não havia sido descrito na literatura para a obtenção de biópsia pulmonar em cães. De certa forma, esta técnica se confronta com os princípios básicos da videocirurgia, citados por Potter \& Hendrickson (1998), em que os portais deveriam ser introduzidos de maneira a conseguir uma triangulação, permitindo melhor manipulação dos instrumentais. No entanto essa disposição dos trocartes não conferiu qualquer limitação para a realização da biópsia, e foi instituída propositalmente para se evitar a necessidade de introdução intercostal. De outra forma, a realização de cirurgia vídeo-endoscópica sem triangulação adequada tem mostrado sucesso em vários procedimentos enquadrados na categoria "LESS" (Laparoendoscopic Single-site Surgery), realidade crescente em medicina (Kommu \& Rané 2009).

A respeito das técnicas toracoscópicas existentes para a obtenção de biópsia pulmonar, destaca-se que muitas delas já são de grande aceitabilidade na medicina humana, no entanto, na medicina veterinária o uso é restrito, sendo raramente relatada. Para tanto, aplica-se principalmente a técnica de ligadura endolloop a partir do acesso com três portais, conforme descrito por Potter \& Hendrickson (1998). No atual experimento foi proposto o uso de apenas dois portais a fim de torná-la ainda menos invasiva. Técnicas de dois portais foram descritas na medicina humana, entretanto, são frequentemente instituídas empregando-se grampeadores cirúrgicos, os quais tendem a aumentar os custos do procedimento (Colt et al. 1995). Embora Rocco et al. (2004), tenham defendido a realização de biópsia pulmonar por CTVA com um único portal, ainda é questionável se tal procedimento é menos invasivo que a técnica de dois portais aqui exposta, pois para a execução, esses autores realizaram uma incisão intercostal, simulando uma mini-toracotomia. Se as amostras a serem coletadas não apresentarem diâmetro elevado, que não permita suas passagens pelo interior da cânula, a técnica paraxifoide ainda permitiria biópsias múltiplas dos diferentes lóbulos pulmonares, o que não poderia ser obtido por CTVA com único acesso.
A indução de pneumotórax previamente à entrada dos portais foi estabelecida com a intenção de distender o diafragma e, principalmente, afastá-lo das estruturas intratorácicas nobres (pulmões, coração e grandes vasos), facilitando a entrada do trocarte e oferecendo menores chances de lesões orgânicas, haja vista que não foi realizada intubação seletiva. A quantidade de ar utilizada ( $30 \mathrm{ml} \mathrm{kg}^{1} /$ hemitórax) foi baseada nas observações obtidas em experimento prévio realizado por Pigatto et al. (2008), no qual se verificou que os animais hígidos manifestaram sinais de descompensação cardio-respiratória e elevação da PVC acima de $10 \mathrm{cmH}_{2} \mathrm{O}$, após a introdução de volume de ar igual ou superior a $50 \mathrm{ml} \mathrm{kg}^{-1}$ por hemitórax. Outro fator que está relacionado à relativa segurança da indução do pneumotórax no presente experimento é o curto período de tempo, no qual os pacientes foram submetidos à alta pressão intratorácica.

Referindo-se à técnica de colheita, o emprego exclusivo da pinça de videocirurgia de biópsia saca-bocado não foi efetivo para o tecido pulmonar, pois permitiu sua apreensão, mas não a subsequente secção. Isso ocorreu, pois tal pinça é utilizada para biópsia de tecido parenquimatoso, e o pulmão, diferentemente, é um órgão composto por tecido conjuntivo rico em fibras elásticas, o que torna mais difícil sua laceração (Bacha Jr. \& Bacha 2003). Dessa forma, a introdução intercostal de uma agulha $40 \times 12 \mathrm{~mm}$ foi importante nessa técnica, pois possibilitou a secção tecidual e a obtenção de materiais para análise histológica.

As amostras obtidas foram consideradas satisfatórias, segundo o laudo histopatológico, sendo possível visibilizar a presença de tecido pulmonar (alvéolos e brônquios) e diagnosticar atelectasia multifocal discreta, moderada e severa, distribuídas entre os diferentes cães. A cicatrização do sítio de biópsia ocorreu por segunda intenção, provavelmente pela deposição de tampão de fibrina no local seccionado, dispensando suturas do parênquima pulmonar.

A presença do endoscópio no primeiro portal permitiu verificar quanto ao correto posicionamento do dreno após a sua introdução pelo segundo portal, eliminando assim a necessidade de exame radiográfico para confirmar tal condição. Embora Pigatto et al. (2008) já tivessem comprovado que o acesso paraxifoide é adequado para a drenagem torácica com tubo em cães, tal trabalho não avaliou adequação da drenagem torácica durante o período pós-operatório, haja vista que não havia risco de escape de ar pelo pulmão e os drenos eram removidos imediatamente após a resolução do pneumotórax induzido. Na presente pesquisa foi possível comprovar a eficácia do drenagem torácica pelo acesso paraxifoide transdiafragmático na presença de lesão pulmonar, mesmo que diminuta, uma vez que esta foi efetiva e sem complicações por até 48h após o procedimento cirúrgico.

Levando em consideração o tamanho do dreno utilizado, apesar da literatura indicar sondas torácicas de 18 a $22 \mathrm{Fr}$ para cães de 7-15 kg (Fossum 2002), optou-se por utilizar dreno de menor calibre que permitisse a passagem no interior do trocarte de $5 \mathrm{~mm}$ de diâmetro, condição aceitável quando o conteúdo a ser drenado for ar (Fossum 2002). A presen- 
ça de sangue no conteúdo drenado nos pacientes desse estudo não conferiu limitação para sua efetiva atuação. Dos 13 animais utilizados para a avaliação da técnica proposta, somente em 12 foram considerados os valores da drenagem torácica, pois um dos cães removeu o dreno no período pósoperatório. Dos 12 cães, onze $(91,67 \%)$ produziram quantidade de ar e/ou líquido inferior a 2,2 $\mathrm{ml} \mathrm{kg}^{-1} \mathrm{dia}^{-1}$, e os drenos foram removidos após 24 horas, valor indicado por Fossum (2002) para a remoção do dreno torácico. Um dos animais $(8,33 \%)$ produziu $4,1 \mathrm{ml} \mathrm{kg}^{-1} \mathrm{dia}^{-1}$, e em virtude disso o dreno foi removido em $48 \mathrm{~h}$, sendo que de $24-48 \mathrm{~h}$ ocorreu apenas a produção de líquido serosanguinolento, que os autores desse trabalho atribuem a uma reação tipo corpo estranho causado pelo próprio dreno.

Ainda em relação à drenagem torácica nas primeiras 24 $\mathrm{h}$, em três cães não houve nenhuma produção de líquido ou ar $(25 \%)$, um animal $(8,3 \%)$ produziu apenas ar, quatro $(33,3 \%)$ somente líquido serosanguinolento, e em outros três (25\%), verificou-se tanto a drenagem de ar como de líquido serosanguinolento. Em apenas um cão $(8,35 \%)$ foi observada drenagem de sangue nas primeiras quatro horas de pós-operatório. Esses dados serviram para ressaltar que há mínimo escape de ar após a remoção de pequeno fragmento pulmonar. Isto já era esperado pelos autores, pois a pinça utilizada promoveu o esmagamento do tecido pulmonar, junto à borda de corte, evitando maior perda de gás.

A toracoscopia intercostal aos 21 dias do pós-operatório, permitiu verificar o adequado selamento dos defeitos diafragmáticos em todos os animais, o que comprova a desnecessidade de suturas diafagmáticas em feridas produzidas por trocartes de $5 \mathrm{~mm}$ (Pigatto et al. 2008), mesmo na presença de dois acessos simultâneos. Verificaram-se aderências do diafragma à membrana mediastínica em cinco cães $(31,25 \%)$ e do sítio de biópsia à membrana mediastínica, em outros seis amimais (37,5\%). Em cinco animais $(31,25 \%)$ não foram encontradas aderências durante a reavaliação. A presença de aderências intratorácicas é indesejável, pois pode dificultar a manipulação orgânica em uma nova intervenção cirúrgica (Gonçalves et al. 1999), contudo a fixação mediastinal já era condição esperada, e que não demonstrou qualquer significado clínico.

\section{CONCLUSÕES}

Nas condições do presente estudo conclui-se que:

A toracoscopia paraxifoide transdiafragmática é adequada para a avaliação bilateral da cavidade torácica e obtenção de biópsia pulmonar em cães, permitindo a aquisição de material para análise de qualquer lobo de ambos os pulmões, e apresentando-se como uma técnica rápida e segura em animais experimentais;

A colheita de material para biópsia com a pinça sacabocado é eficiente quando associada à utilização de aguIha 40/12 para a secção tecidual e a lesão decorrente cicatriza sem necessidade de suturas;

A técnica proposta deve ser realizada preferencialmente com ventilação controlada, com a finalidade de evitar o desenvolvimento de acidose respiratória.
Agradecimentos.- À empresa Exatech Indústria e Comércio Ltda pelo empréstimo da pinça endoscópica saca bocado Edlo.

\section{REFERÊNCIAS}

Andreasen C.B. 2003. Bronchoalveolar lavage. Vet. Clin. North Am., Small Anim. Pract. 33:69-88.

Aguiar E.S.V., Dallabrida A.L., Bopp S., Rocha G.L.S., França E.P., Fonseca E.T., Dalmolin F., Demori G., Silva J.H.S. \& Raiser A.G. 2004. Mensuração da pressão venosa central por meio de cateteres venoso central e periférico: comparação entre os valores obtidos em cães e elaboração de índice de correção. Ciência Rural 34:1827-1831.

Bacha Jr.W.J. \& Bacha L.M. 2003. Atlas Colorido de Histologia Veterinária. Roca, São Paulo, p.293-334.

Barreto S.S.M., Filgueiras N., Crespo A.S., Goes A., Vianna A., CarvaIho A., Fernandes E.O., Veras J., Castro J.E., Filguerias N., CarvaIho P.R.A., Plutinik R. \& Vieir S.R.R. 2000. Indicações de ventilação mecânica invasiva com pressão positiva. J. Pneumol. 26(Supl.2): 3-5.

Bomback D.A. 2007. Video-assisted thoracoscopic surgery compared with thoracotomy: Early and late follow-up of radiographical and functional outcome. Spine Journal 7:399-405.

Boutin C., Viallat J.R., Cargnino P. \& Rey F. 1982. Thoracoscopic lung biopsy: Experimental and clinical preliminary study. Chest 82:4448.

Colt H.G., Russack V., Shanks T.G. \& Moser K.M. 1995. Comparison of wedge to forceps videothoracoscopic lung biopsy. Chest 107:546550.

Fossum T.W. 2002. Cirurgia de Pequenos Animais. Roca, São Paulo, p.752-785.

Gonçalves R.M., Esquerdo C.R.M., Petroianu A. \& Barbosa A.J.A. 1999. Influência de aderências peritoneais e fio cirúrgico na tensão de ruptura da parede abdominal em ratos. Revta Colégio Bras. Cirurg. 27:147-152.

Harris R.J., Kavuru M.S., Rice T.W. \& Kirby T.J. 1995. The diagnostic and therapeutic utility of thoracoscopy. Chest 108:828-841.

Hendrix P.K. \& Raffe M.R. 1998. Distúrbios dos líquidos, eletrólitos e ácidobásicos, p.26-28. In: Bojrab M.J. (Ed.), Mecanismos da Moléstia na Cirurgia dos Pequenos Animais. Manole, São Paulo.

Hopper K. \& Haskins S.C. 2008. A case-based review of a simplified quantitative approach to acid-base analysis. J. Vet. Emerg. Critical Care 18:467-476.

Kommu S.S. \& Rané A. 2009. Devices for laparoendoscopic singlesite surgery in urology. Expert Rev. Med. Devices 6:95-103.

Miller C.J. 2007. Approach to the respiratory patient. North Am. Vet. Clin. Small Anim. Pract. 37:861-878.

Norris C.R., Griffey S.M., Samii V.F., Cristopher M.M. \& Mellema M.S. 2002. Thoracic radiography, bronchoalveolar lavage cytopathology, and pulmonary parenchymal histopathology: A comparison of diagnostic results in 11 cats. J. Am. Vet. Anim. Hospital Assoc. 38:337345.

Ochoa L.N. 2005. Acid-base principles and practical interpretation in small animals. Proc. World Small Animal Veterinary Association World Congress, México. Disponível em: http://www.vin.com? members/Proceedings/Proceedings.plx Acesso em 28 de novembro de 2005.

Pigatto J., Brun M.V., Barcellos L.J.G., Rausch S.F., Phol V.H., Feranti J.P.S. \& Guedes R.L. 2008. Produção de pneumotórax em cães e manejo por toracoscopia paraxifoide transdiafragmática. Ciência Rural 38:2210-2217.

Potter L. \& Hendrickson D.A. 1998. Therapeutic video-assisted thoracic surgery, p.169-187. In: Freeman L.J. (Ed.), Veterinary Endosurgery. Mosby, St Louis. 
Reineiro C.R. \& Cohn L.A. 2007. Interstitial lung diseases. North Am. Vet. Clin., Small Anim.Pract. 37:937-947.

Rena O., Casadio C., Leo F., Giobbe R., Cianci R., Baldi S., Rapellino M. \& Maggi G. 1999. Videothoracoscopic lung biopsy in the diagnosis of interstitial lung disease. Eur. J. Cardio-Thoracic Surg. 16:624-627.

Rocco G., Martin-Ucar A. \& Passera E. 2004. Uniportal VATS wedge pulmonary resections. Annals Thoracic Surg. 77:726-728.
Twedt D.C. 2002. Diagnostic toracoscopy. Western Veterinary Conference, Colorado. Disponível em http://www.vin.com/Members/ Proceedings/Proceedings.plx Acesso em 9 de outubro de 2006.

Zoppa A.L.V., Alvarenga J., Cruz R.S.F., Machado T.S.L. \& Silva L.C.L.C. 2008. Toracoscopia aplicada à ressecção de fragmento pulmonar com auxílio de sutura mecânica em equinos. Arq. Bras. Med. Vet. Zootec. 60:559-565. 